\title{
Correction to: Dynamic Response Analysis of Large Arch-Roof Oil Tank Subjected to the Coupling Impact of Two-Source Blast Waves Based on Finite Element Method
}

\author{
Guohua Chen · Fan Wang • Chilou Zhou $\cdot$ Kun Hu
}

Published online: 7 February 2020

(C) ASM International 2020

Correction to: J Fail. Anal. and Preven. https://doi.org/10.1007/s11668-020-00833-w

In the original version of the article, some of the entries in Table 12 were misaligned. Following is the corrected table:
The original article has been corrected.

Table 12 Dynamic response state of the tank wall in $0^{\circ}, 30^{\circ}$ direction

\begin{tabular}{|c|c|c|c|c|c|c|c|c|}
\hline \multirow[b]{2}{*}{$\begin{array}{l}\text { Off-ground } \\
\text { height }(\mathrm{m})\end{array}$} & \multicolumn{3}{|c|}{$0^{\circ}$ direction } & \multirow[b]{2}{*}{ Response state } & \multicolumn{3}{|c|}{$30^{\circ}$ direction } & \multirow[b]{2}{*}{$\begin{array}{l}\text { Response } \\
\text { state }\end{array}$} \\
\hline & $\begin{array}{c}\text { Maximum } \\
\text { stress (MPa) }\end{array}$ & $\begin{array}{l}\text { Dynamic yield } \\
\text { strength (MPa) }\end{array}$ & Difference & & $\begin{array}{c}\text { Maximum } \\
\text { stress (MPa) }\end{array}$ & $\begin{array}{l}\text { Dynamic yield } \\
\text { strength (MPa) }\end{array}$ & Difference & \\
\hline 0.242 & 332 & 391.9 & -59.9 & Elastic deformation & 325 & 391.9 & -66.9 & Elastic deformation \\
\hline 2.098 & 360 & & -31.9 & & 334 & & -57.9 & \\
\hline 4.419 & 330 & & -61.9 & & 320 & & -71.9 & \\
\hline 6.739 & 326 & & -65.9 & & 322 & & -69.9 & \\
\hline 9.060 & 357 & & -34.9 & & 328 & & -63.9 & \\
\hline 11.38 & 498 & & 106.1 & Yield failure & 474 & & 82.1 & Yield failure \\
\hline 13.70 & 631 & & 239.1 & & 545 & & 153.1 & \\
\hline 16.02 & 499 & & 107.1 & & 531 & & 139.1 & \\
\hline
\end{tabular}

The original article can be found online at https://doi.org/10.1007/ s11668-020-00833-w.

G. Chen $\cdot$ F. Wang $\cdot$ C. Zhou $(\bowtie) \cdot$ K. Hu

School of Mechanical and Automotive Engineering, South China

University of Technology, Guangzhou 510641, Guangdong,

People's Republic of China

e-mail: mezcl@scut.edu.cn; Chilou.Zhou@warwick.ac.uk

G. Chen $\cdot$ F. Wang $\cdot$ C. Zhou $\cdot$ K. Hu

Guangdong Provincial Science and Technology Collaborative Innovation Center for Work Safety, Guangzhou 510641,

Guangdong, People's Republic of China
Publisher's Note Springer Nature remains neutral with regard to jurisdictional claims in published maps and institutional affiliations.

C. Zhou

School of Engineering, University of Warwick, Coventry CV4

7AL, UK 